\section{Retinal abnormalities in multiple sclerosis patients with associated chronic cerebrospinal venous insufficiency}

\author{
Aneta Adamczyk-Ludyga, ${ }^{1}$ \\ Justyna Wróbeł, ${ }^{1}$ Marian Simka, ${ }^{2}$ \\ Tomasz Ludyga, ${ }^{2}$ Paweł Latacz, \\ Marek Kazibudzki
}

'Euromedic Specialist Clinics, Department of Ophthalmology, Katowice, Poland;

${ }^{2}$ Euromedic Specialist Clinics, Department of Vascular \& Endovascular Surgery, Katowice, Poland

\section{Abstract}

Optical coherence tomography (OCT) is a non-invasive method for the assessment of optic nerve fibers and retinal ganglion cells. This study was aimed at the assessment of retinal abnormalities in multiple sclerosis patients in the context of chronic cerebrospinal venous insufficiency using 0CT of the retina and the optic nerve. We examined 239 multiple sclerosis (MS) patients, including 220 patients with associated chronic cerebrospinal venous insufficiency and 19 MS patients without venous pathology. The following OCT parameters were assessed: average ganglion cell complex thickness, global loss volume, focal loss volume and average retinal nerve fibre layer thickness. Abnormalities in the azygous and internal jugular veins were evaluated using catheter venography. We found a higher prevalence of abnormal OCT parameters in the patients with previous history of optic neuritis, not only on the side of inflammatory event, but also in the contralateral eye, which is in line with already existing body of evidence. The new and intriguing discovery is that we found statistically significant higher prevalence of abnormal OCT values in multiple sclerosis patients with unilateral stenosis of internal jugular vein. Patients who were not found venous abnormalities, as well as those presenting with pathologic azygous or bilateral internal jugular venous outflows, did not demonstrate a changed frequency of abnormal OCT parameters. Potential association between venous malformations and eye manifestations of multiple sclerosis, as has been demonstrated in this report, justifies further studies on this topic.

\section{Introduction}

Optical coherence tomography (OCT) uses light interference patterns to make a cross-section image of the layers of retina and intraocular portion of the optic nerve. In this way OCT is capable of assessment of the retinal ganglion cells and their unmyelinated axons as they converge on the optic disc. This diagnostic tool provides precise and reproducible information on these structures in different pathologies affecting the anterior visual pathway, including also multiple sclerosis (MS). ${ }^{1-3}$ Axonal loss within the anterior optic pathway in MS patients can be detected not only after an episode of optic neuritis $(\mathrm{ON})$, one of the most common manifestations of MS, but also in the patients without a history of $\mathrm{ON}$, thus reflecting a disperse MS-associated neurodegeneration. ${ }^{4-7}$ It has recently been described that majority of MS patients present with the so-called chronic cerebrospinal venous insufficiency (CCSVI), vascular pathology comprising venous malformations in the main veins draining the central nervous system. ${ }^{8,9}$ It this paper we present the results of study that evaluated relations between 0CT findings and venous outflow abnormalities in MS patients. We found that unilateral stenosis of the internal jugular vein (IJV), a subtype of CCSVI, was associated with an increased prevalence of abnormal OCT values.

\section{Materials and Methods}

This ophthalmological study was a part of clinical trial on endovascular treatments for CCSVI in MS patients. The entire study was designed to assess safety and efficacy of endovascular procedures performed to alleviate venous outflow blockages in the main veins draining the central nervous system in these patients. The study was approved by the Bioethical Committee of the Regional Silesian Board of Physicians in Katowice, Poland (approval No: 7/2010) and was registered at ClinicalTrials.gov; identifier: NCT01264848. All patients provided their written consent to undergo the procedures and diagnostic tests.

OCT of the eyes was one of pre-procedural non-invasive tests. Primary aim of this examination was to evaluate MS-related damage to the retina and optic nerves, and also to assess these abnormalities at follow-ups after endovascular treatments for CCSVI. In this paper we present OCT findings before endovascular treatment. There were assessed 239 patients (476 eyes examined) with clinically defined MS, 168 women and 71 men. The patients were aged 17-69 years, with a median age of 43 years. They suffered from MS for 0.5 to 40 years, with a median duration of the dis-
Correspondence: Marian Simka, ul. Jednosci 20 , 43-245 Studzionka, Poland.

Tel. +48.322.120.498.

E-mail: mariansimka@poczta.onet.pl

Key words: multiple sclerosis, optic nerve, optical coherence tomography, retina, venous insufficiency.

Contributions: AA-L, JW, MS, TL, PL, MK, conception and design, article revising and final approval; AA-L, JW, interpretation of data, ophthalmological part of the study; MS, collection and interpretation of data, statistical analysis; TL, PL, MK, endovascular part of the study.

Conflict of interests: all authors are employed in the hospital, where the treatments for CCSVI are patient-paid; AA-L her family member is the coowner of hospital where the treatments for CCSVI are patient-paid; MS received publication fees from Servier International and speaker fees from American Access Care; received congress costs reimbursement from Esaote International; TL is the owner of patent on stent design that potentially could be used for the treatment of venous lesions (the stent is not yet available in the market); his family member is the co-owner of hospital where the treatments for CCSVI are patient-paid.

Acknowledgements: the paper has been presented at $2^{\text {nd }}$ Annual Meeting of International Society of Neurovascular Disease, Orlando, USA, 18-22 ${ }^{\text {nd }}$ February 2012 and at $14^{\text {th }}$ Annual Meeting of Australasian College of Phlebology, Melbourne, Australia, $30^{\text {th }}$ March-3 $3^{\text {rd }}$ April 2011.

Received for publication: 14 March 2012.

Revision received: 21 May 2012.

Accepted for publication: 31 May 2012.

This work is licensed under a Creative Commons Attribution 3.0 License (by-nc 3.0).

(C) Copyright A. Adamczyk-Ludyga et al., 2012

Licensee PAGEPress, Italy

Veins and Lymphatics 2012; 1:e2

doi:10.4081/vl.2012.e2

ease of 10 years. Reliable data on history of $\mathrm{ON}$ were available in 180 patients: 103 patients presented with positive history of $0 \mathrm{~N}, 57$ on one side (23 of right optic nerve and 34 on the left side) and 46 bilaterally. OCT examination was performed on RT100-2 OCT scanner (Optoview Inc., Fremont, CA, USA). The following OCT parameters were assessed: i. average ganglion cell complex thickness (aGCC) in the macula; ii. global loss volume (GLV) of ganglion cell complex; iii. focal loss volume (FLV) of ganglion cell complex; iv. average retinal nerve fibre layer thickness (aRNFL).

The findings were interpreted as: normal, borderline or pathological, according to the built-in software, which compared value of each OCT parameter to the database coming 
from healthy population, after adjusting for the age, sex and ethnicity.

Hemodynamic and/or structural abnormalities in the IJVs, brachiocephalic and azygous veins were evaluated using catheter venography. In this study we did not include the patients with - rather infrequently seen - outflow blockages in the brachiocephalic veins, since these lesions actually represent tandem stenoses (together with coexisting lesions in the IJVs), making difficult an unequivocal interpretation of the results. The details of the venographic protocol for the assessment of examined veins can be found in our previous paper. ${ }^{10}$ The following venographic flow patterns were regarded abnormal and were categorised into four grades: i. grade 1: venous outflow slowed down, no reflux detected; ii. grade 2: venous outflow slowed down, mild reflux and/or pre-stenotic dilation of the vein; iii. grade 3: venous outflow slowed down, with reflux and outflow through collaterals; iv. grade 4: no outflow through the vein, huge outflow through collaterals.

\section{Statistical analysis}

The $\chi^{2}$ test was used to test the null hypothesis that the frequencies of particular OCT parameter were equal, regardless of the distribution of venous lesions, against the alternative hypothesis that these frequencies differed significantly between patients' subgroups. The samples were compared to average OCT results in CCSVI-positive patients. Analysis was performed using PAST data analysis package (version 2.09; University of Oslo, Norway). Significance of $\mathrm{P}$ values was set at $\mathrm{P}<0.05$.

\section{Results}

\section{Pathological optical coherence tomography parameters}

Abnormal aGCC values were found in $46.2 \%$ and borderline values in $12.4 \%$ of MS patients; GLV values - in $57.6 \%$ and $9.9 \%$; FLV values - in $52.6 \%$ and $7.2 \%$; aRNFL values - in $43.7 \%$ and $18.2 \%$, respectively.

\section{Pathological venographic findings}

Venous outflow abnormalities in at least one vein draining the central nervous system (left or right IJV, or the azygous vein) were found in 220 cases ( $92.1 \%$ of the patients). Lesions in one vein were found in 78 patients $(32.6 \%)$, in two veins: 131 patients (54.8\%) and in three veins: 11 patients (4.6\%). Most of the lesions were found in the IJVs, only two patients presented with isolated stenoses of the azygous vein. Outflow abnormalities of the azygous vein, isolated or in combination with IJV lesions, were found in 17 patients (7.1\%).

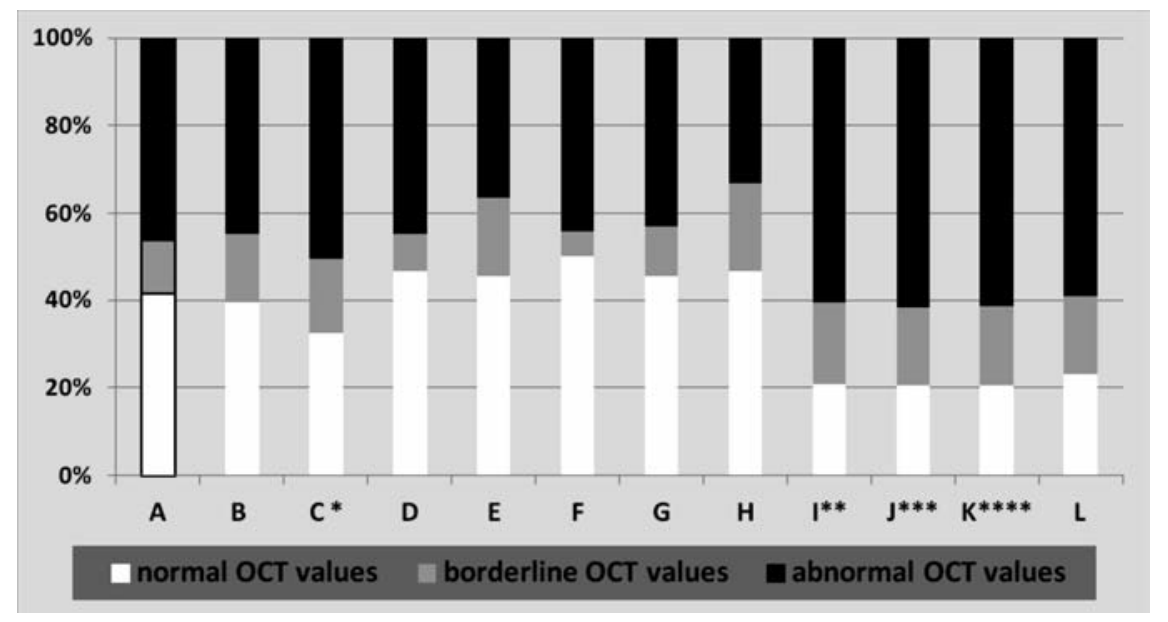

Figure 1. Optical coherence tomography (OCT) findings and venous abnormalities: average ganglion cell complex thickness: $\mathrm{A}$, average findings [chronic cerebrospinal venous insufficiency (CCSVI) patients]; B, no CCSVI detected; C, one pathological vein $\left({ }^{*} \mathrm{P}=0.04\right)$; $\mathrm{D}$, two pathological veins; $\mathrm{E}$, three pathological veins; $\mathrm{F}$, pathological azygous vein; $G$, the only affected vein: $C C S V I$ score $=1 ; H$, the only affected vein: $C C S V I$ score $=2$; I, the only affected vein: CCSVI score $=3(* * P=0.01)$; J, the only affected vein: CCSVI score $=4\left({ }^{* * *} \mathrm{P}=0.046\right)$; $\mathrm{K}$, unilateral stenosis of internal jugular vein (IJV) (grade 3 or 4 ) ipsilateral eye $\left({ }^{* * * *} \mathrm{P}=0.03\right)$; $\mathrm{L}$, unilateral stenosis of IJV (grade 3 or 4 ) - contralateral eye $(\mathbf{P}=\mathbf{0 . 0 5 5})$.

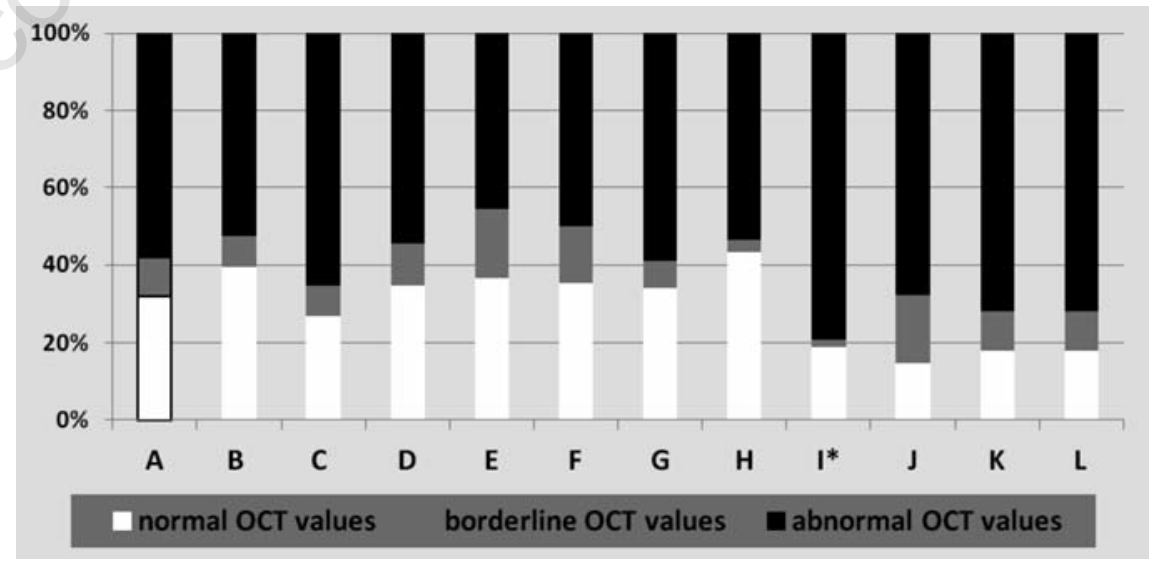

Figure 2. Optical coherence tomography (OCT) findings and venous abnormalities: global loss volume of ganglion cell complex: A, average findings [chronic cerebrospinal venous insufficiency (CCSVI) patients]; B, no CCSVI detected; C, one pathological vein; D, two pathological veins; $E$, three pathological veins; F, pathological azygous vein; $G$, the most affected vein: CCSVI score $=1 ; H$, the most affected vein: CCSVI score=2; $I$, the most affected vein: CCSVI score $=3$; J, the most affected vein: $C C S V I$ score $=4$; $K$, the only affected vein: CCSVI score $=1$; $L$, the only affected vein: CCSVI score $=2 ; M$, the only affected vein: $C$ CSVI score $=3\left({ }^{*} \mathrm{P}=0.01\right)$; $\mathrm{N}$, the only affected vein: $\mathrm{CCSVI}$ score $=4(\mathrm{P}=0.06) ; \mathrm{O}$, unilateral stenosis of internal jugular vein (IJV) (grade 3 or 4) - ipsilateral eye; P, unilateral stenosis of IJV (grade 3 or 4 ) - contralateral eye. 


\section{Correlations between optical coherence tomography findings and optic neuritis history}

We found a much higher prevalence of abnormal OCT parameters in the patients with previous history of $\mathrm{ON}$, not only on the side of such an inflammatory event, but also in the contralateral eye. These differences were statistically significant if compared to the frequency of this ocular pathology in the patients with negative $\mathrm{ON}$ history (details not shown).

\section{Correlations between optical coherence tomography findings and venographic patterns of chronic cerebrospinal venous insufficiency}

We found a higher, statistically significant prevalence of abnormal OCT parameters: aGCC, FLV and aRNFL in the patients with unilateral stenosis of IJV, and also a trend towards such an increased frequency of pathologic GLV values. Looking into details, it could be seen that especially severe (grade 3 and 4 ) unilateral lesions were associated with increased prevalence of pathological OCT values, while the patients with mild-degree lesions exhibited less frequent ocular abnormalities. Although abnormal OCT parameters were primarily found in the eyes ipsilaterally to high grade stenoses of the IJV, they were also seen - even if this tendency was less pronounced - in the eyes contralaterally to such a stenosis. CCSVI-negative patients, as well as those presenting with bilateral IJV stenoses, or azygous vein pathology, demonstrated a similar prevalence of abnormal OCT results. Only in the case of the FLV, CCSVI-negative patients exhibited a trend towards less frequent OCT abnormalities. The details of OCT findings are presented in Figures 1-4.

\section{Discussion}

Many authors, using OCT techniques, have already described the signs of loss of retinal ganglion cells in MS patients. Damage to these neurons was traditionally thought to be a result of $0 N$. Indeed, frequency of retinal pathology measured with OCT technology is more common in the cases with previous $0 \mathrm{~N}$ and our findings are in line with these studies. ${ }^{6,7}$ Still, we also revealed that abnormal 0CT values were more common in MS patients with unilateral blockage of the IJV. Still, we were unable to demonstrate significantly changed prevalence of abnormal OCT values in MS patients presenting with other anatomical variants of CCSVI (bilateral stenoses of the IJVs, lesions in the azygous vein) or in the patients with no venous pathology detected.

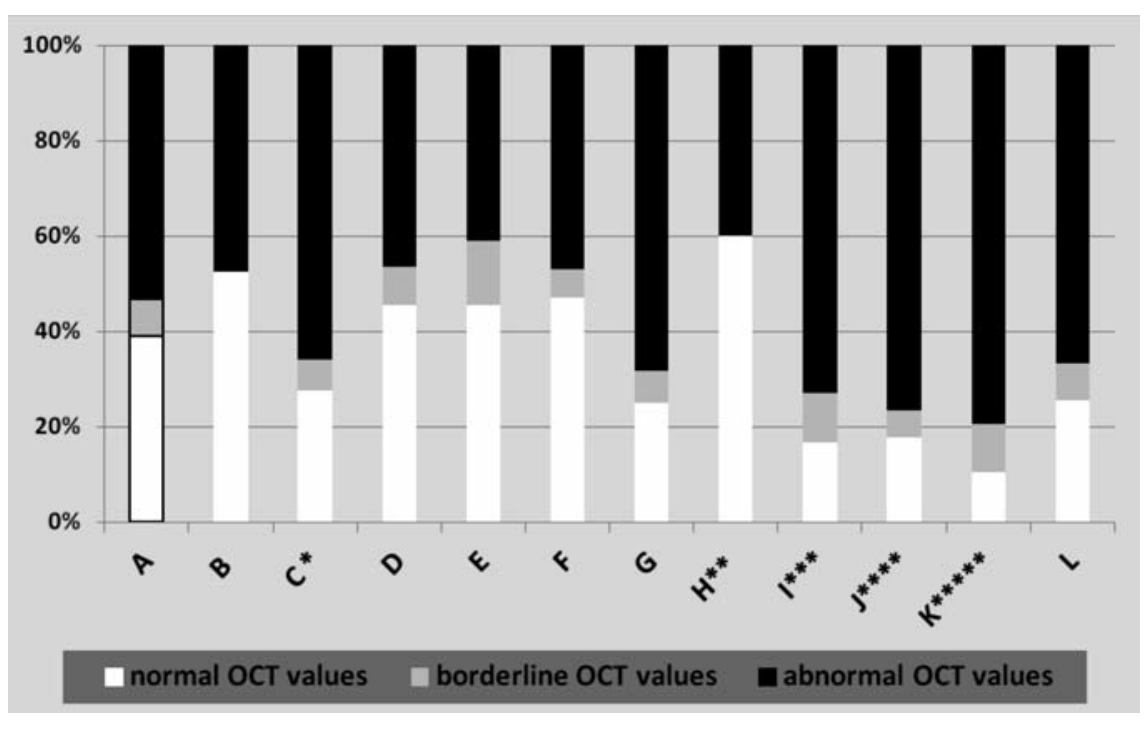

Figure 3. Optical coherence tomography (OCT) findings and venous abnormalities: focal loss volume of ganglion cell complex: $\mathrm{A}$, average findings [chronic cerebrospinal venous insufficiency (CCSVI) patients]; B, no CCSVI detected; C, one pathological vein $\left({ }^{*} P=0.005\right) ; D$, two pathological veins; $E$, three pathological veins; $F$, pathological azygous vein; $G$, the only affected vein: $C C S V I$ score $=1 ; H$, the only affected vein: CCSVI score $=2$ $\left({ }^{* *} \mathbf{P}=0.04\right.$ - ocular abnormalities less frequent); I, the only affected vein: CCSVI score $=3$ (*** $\mathrm{P}=0.006)$; $\mathrm{J}$, the only affected vein: $\mathrm{CCSVI}$ score $=4\left({ }^{* * * *} \mathrm{P}=0.02\right) ; \mathrm{K}$, unilateral stenosis of internal jugular vein (IJV) (grade 3 or 4 ) - ipsilateral eye $(* * * * * P=0.001)$; $\mathrm{L}$, unilateral stenosis of IJV (grade 3 or 4 ) - contralateral eye.

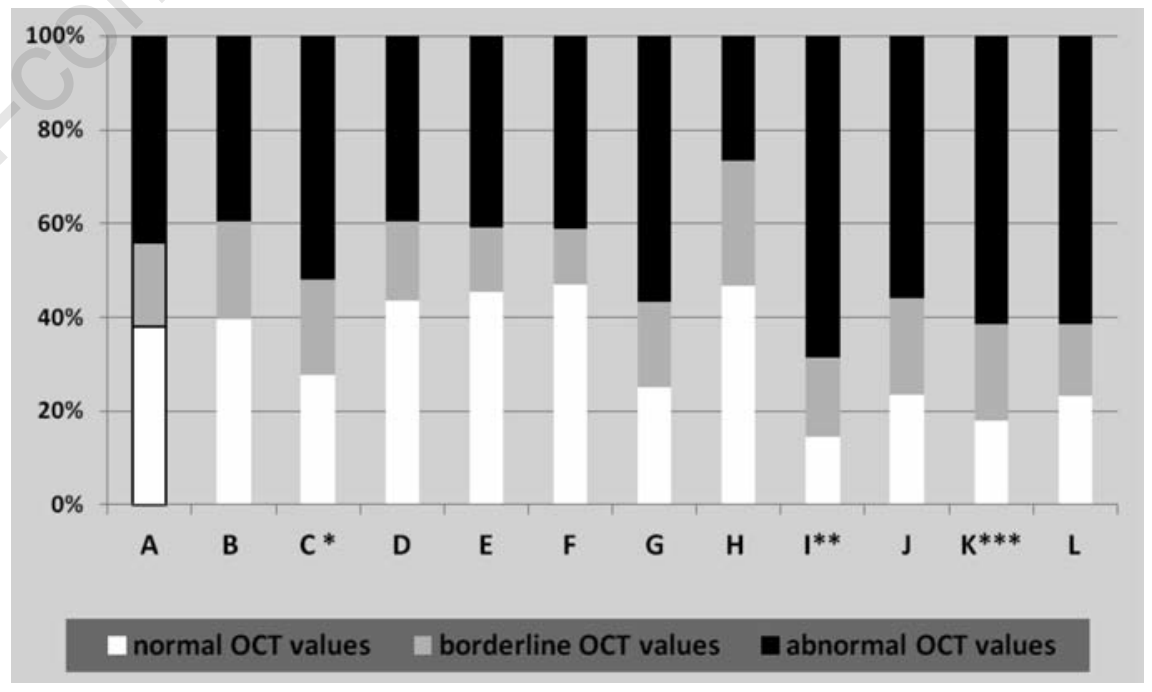

Figure 4. Optical coherence tomography (OCT) findings and venous abnormalities: average retinal nerve fiber layer thickness: A, average findings [chronic cerebrospinal venous insufficiency (CCSVI) patients]; B, no CCSVI detected; C, one pathological vein ( $\mathrm{P}=\mathbf{0 . 0 3}$ ); $\mathrm{D}$, two pathological veins; $\mathrm{E}$, three pathological veins; $\mathrm{F}$, pathological azygous vein; $G$, the only affected vein: $C C S V I$ score $=1 ; H$, the only affected vein: $C C S V I$ score $=2$; I, the only affected vein: CCSVI score $=3\left({ }^{* *} \mathrm{P}=0.001\right)$; $\mathrm{J}$, the only affected vein: CCSVI score $=4$; $K$, unilateral stenosis of internal jugular vein (IJV) (grade 3 or 4) - ipsilateral eye $(* * * \mathbf{P}=0.03)$; $\mathrm{L}$, unilateral stenosis of IJV (grade 3 or 4$)$ - contralateral eye. 
These findings suggest that extracranial venous abnormality can contribute to neurological pathology in the settings of MS.

At the moment it is difficult to explain our findings unequivocally. To the best of our knowledge it is the first study looking at potential impact of venous lesions on retinal abnormalities in MS. In order to reveal a potential link between CCSVI and MS, we looked at correlations between two different objective tests: OCT of the retina and catheter venography of the azygous and IJVs. Although both tests have established roles in current medical diagnostics and are considered reliable, there are also several limitations that could challenge final conclusions coming from our study. OCT has been shown to be a reproducible imaging technique, still a number of procedural and biological factors might influence the results. These include: operator-related artifacts (e.g., defocusing and depolarisation errors), poor patients cooperation, concomitant eye pathologies (e.g., cataract, retinal drusen), age and ethnicity of the patients. Most likely, we were unable to avoid at least some of these errors. Moreover, which of the OCT parameters should be used for the assessment of MS-related damage of the anterior visual pathway is still the matter of debate. Therefore, our results, even statistically significant, should be interpreted with caution.

Catheter venography is regarded as a gold standard for the assessment of pathologies of large veins. It is far more reliable and much less operator-dependent than other tests evaluating venous flow and anatomy. Still, the use of catheter venography in this study is somewhat weakened by the fact that assessment of the flow in azygous and jugular veins is new field of expertise. Consequently, diagnostic accuracy of this test was probably far from perfect. In addition, our scale of CCSVI grading ${ }^{10}$ is not yet widely accepted.

Although most of the current scientific research on MS is focused on immune processes, a potential link between MS and the veins has been well known for decades. For example, in 1935 Putnam demonstrated MSlike cerebral plaques in the dogs with artificially obstructed cerebral venous outflow, ${ }^{11}$ while retinal periphlebitis, common eye manifestation of MS, was described for the first time in 1947. ${ }^{12}$ Theoretically, ophthalmic veins can be vulnerable in the case of disturbed blood flow, since they connect the intra- and extracranial venous networks and can be overloaded if extracranial portion of the IJV vein becomes obstructed. Disturbed blood-brain barrier (BBB) can be the other source of injury to the retina and optic nerves in the settings of disturbed venous outflow. Disintegration of BBB is a hallmark of MS. Such a dysfunctional BBB is not necessarily an effect of inflammation, but may also be triggered by pathologic venous circulation. ${ }^{13}$ Interestingly, it is known that even at physiologic settings microvessels of the prelaminar optic nerve head lack the BBB characteristics. ${ }^{14}$ We found an increased prevalence of OCT abnormalities in MS patients with severe unilateral obstruction of the IJV, while the patients with bilateral blockages presented with a less frequent eye pathology. Although one might expected a higher rate of retinal pathology in the patients with more numerous venous lesions, actually - from anatomical and physiological point of view unilateral occlusion of the IJV can be more deleterious for the eye. In such a case venous outflow from the brain can be preferentially shifted via ophthalmic veins towards external jugular vein system, resulting in the overload of veins draining the eye and optic nerves. Indeed, in some MS patients we have observed this outflow pattern during venography. On the other hand, an obstructed azygous vein is unlikely to impair ocular circulation. Thus, an unchanged prevalence of retinal pathology in MS patients with azygous vein involvement is in line with these theoretical conjectures. Interestingly, in one study MS patients with benign clinical course of the disease were found more pronounced retinal nerve fibre layer thinning. ${ }^{15}$ Although at the moment no strong evidence exists for a correlation between the severity of venous blockages and more aggressive course of MS, some preliminary observations indicate that such a relationship may exist. ${ }^{16}$ Consequently, the patients with unilateral IJV stenosis seem to be more likely to exhibit a benign clinical course of MS. Interestingly, in our patient series an unilateral $\mathrm{ON}$ was seen more often on the left side. A similar slightly higher frequency of $\mathrm{ON}$ on the left side has already been reported. ${ }^{17,18}$ This unequal distribution of $\mathrm{ON}$ seems to mirror distribution of venous abnormalities in MS patients, since the stenoses are primarily found in the left IJV..$^{19,20}$

Discovery of venous pathologies accompanying MS - a risk factor for injury to the retinal ganglion cells - may solve some puzzles related to this disease. MS patients very often exhibit ocular pathology, even with no previous history of ON. Therefore, it could be suspected that MS-related neurodegeneration, and not $\mathrm{ON}$, is primarily responsible for this injury. However, longitudinal studies of the retinal nerve fibre layer did not reveal a progression of retinal abnormalities in MS patients. ${ }^{21,22}$ Also, histological evaluation of the eyes from autopsied patients demonstrated only an insignificant trend towards association of retinal atrophy with the duration of MS. ${ }^{23}$ The only reasonable explanation of this puzzle may be that: either only the first, even subclinical, attack of $\mathrm{ON}$ results in retinal ganglion cell loss (which seems not very credible), or that retinal abnormalities develop well before clinically overt symptoms of MS. Currently CCSVI is thought to be a congenital pathology. ${ }^{24,25}$ Thus, if these eye pathologies were related to a congenital venous malformation, one should expect that injury to the retina develops much earlier, even in the childhood, which would explain the inconsistencies coming from the above-citied studies.

\section{Conclusions}

A potential association between CCSVI and MS, including eye manifestations of MS, as has been demonstrated in this report, justifies further studies on this topic. Such a research can bring to light pathomechanisms responsible for retinal damage related to MS and perhaps also other diseases of the eye.

\section{References}

1. Noval S, Contreras I, Muñoz S, et al. Optical coherence tomography in multiple sclerosis and neuromyelitis optica: an update. Mult Scler Int 2011;2011:472790.

2. Zaveri MS, Conger A, Salter A, et al. Retinal imaging by laser polarimetry and optical coherence tomography evidence of axonal degeneration in multiple sclerosis. Arch Neurol 2008;6:924-8.

3. Cettomai D, Pulicken M, Gordon-Lipkin E, et al. Reproducibility of optical coherence tomography in multiple sclerosis. Arch Neurol 2008;65:1218-22.

4. Henderson APD, Trip SA, Schlottmann PG, et al. An investigation of the retinal nerve fibre layer in progressive multiple sclerosis using optical coherence tomography. Brain 2008;131:277-87.

5. Jeanjean L, Castelnovo G, Carlander B, et al. Retinal atrophy using optical coherence tomography (OCT) in 15 patients with multiple sclerosis and comparison with healthy subjects. Rev Neurol (Paris) 2008; 164:927-34

6. Davies EC, Galetta KM, Sackel DJ, et al. Retinal ganglion cell layer volumetric assessment by spectral-domain optical coherence tomography in multiple sclerosis: application of a high-precision manual estimation technique. J Neuroophthalmol 2011;31:260-4.

7. Bock M, Brandt AU, Dörr J, et al. Patterns of retinal nerve fiber layer loss in multiple sclerosis patients with or without optic neuritis and glaucoma patients. Clin Neurol Neurosurg 2010;112:647-52.

8. Zamboni P, Galeotti R, Menegatti E, et al. Chronic cerebrospinal venous insufficiency in patients with multiple sclerosis. J Neurol Neurosurg Psychiatry 2009;80:392-9.

9. Zamboni P, Consorti G, Galeotti R, et al. 
Venous collateral circulation of the extracranial cerebrospinal outflow routes. Curr Neurovasc Res 2009;6:204-2.

10. Ludyga T, Kazibudzki M, Simka M, et al. Endovascular treatment for chronic cerebrospinal venous insufficiency: is the procedure safe? Phlebology 2010;25:286-95.

11. Putnam T. Studies in multiple sclerosis: encephalitis and sclerotic plaques produced by venular obstruction. Arch Neurol Psychiatry 1935;33:929-40.

12. Rucker CW. Retinopathy of multiple sclerosis. Trans Am Ophthalmol Soc 1947;45: 564-70.

13. Simka M. Blood brain barrier compromise with endothelial inflammation may lead to autoimmune loss of myelin during multiple sclerosis. Curr Neurovasc Res 2009; 6:132-9.

14. Hofman P, Hoyng P, van der Werf F, et al. Lack of blood-brain barrier properties in microvessels of the prelaminar optic nerve head. Invest Ophthalmol Vis Sci 2001;42: 895-901.

15. Galetta KM, Talman LS, Lile DJ, et al. Visual pathway axonal loss in benign mul- tiple sclerosis [abstract]. Mult Scler 2010: 16 Suppl 10:S23-4.

16. Zamboni P, Menegatti E, WeinstockGuttman B, et al. Hypoperfusion of brain parenchyma is associated with the severity of chronic cerebrospinal venous insufficiency in patients with multiple sclerosis: a cross-sectional preliminary report. BMC Med 2011;9:22.

17. Bradley WG, Whitty CW. Acute optic neuritis: its clinical features and their relation to prognosis for recovery of vision. $\mathrm{J}$ Neurol Neurosurg Psychiatry 1967;30:5318.

18. Bradley WG, Whitty CW. Acute optic neuritis: prognosis for development of multiple sclerosis. J Neurol Neurosurg Psychiatry 1968;31:10-8.

19. Simka M, Latacz $P$, Ludyga $T$, et al. Prevalence of extracranial venous abnormalities: results from a sample of $586 \mathrm{mul}-$ tiple sclerosis patients. Funct Neurol 2011; 26:197-203.

20. Petrov I, Grozdinski L, Kaninski G, et al. Safety profile of endovascular treatment for chronic cerebrospinal venous insuffi- ciency in patients with multiple sclerosis. J Endovasc Ther 2011;18:314-23.

21. Serbecic N, Aboul-Enein F, Beutelspacher $\mathrm{SC}$, et al. High resolution spectral domain optical coherence tomography (SD-OCT) in multiple sclerosis: the first follow up study over two years. PloS ONE 2011; 6:e19843.

22. Henderson AP, Trip SA, Schlottmann PG, et al. A preliminary longitudinal study of the retinal nerve fiber layer in progressive multiple sclerosis. J Neurol 2010;257:1083-91.

23. Green AJ, McQuaid S, Hauser SL, et al. Ocular pathology in multiple sclerosis: retinal atrophy and inflammation irrespective of disease duration. Brain 2010;133: 1591-601.

24. Lee BB, Bergan J, Gloviczki P, et al. Diagnosis and treatment of venous malformations. Consensus Document of the International Union of Phlebology (IUP)2009. Int Angiol 2009;28:434-51.

25. Lee BB, Laredo J, Neville R. Embryological background of truncular venous malformation in the extracranial venous pathways as the cause of chronic cerebrospinal venous insufficiency. Int Angiol 2010;29:95-108. 